\title{
A Comparison of Different Modeling Strategies for Predicting Effective Properties of 3D Woven Composites
}

\author{
Trenton M. Ricks ${ }^{1}$ \\ NASA Glenn Research Center, Cleveland, OH, 44135, United States of America \\ Babak Farrokh ${ }^{2}$ \\ NASA Goddard Space Flight Center, Greenbelt, MD, 20771, United States of America \\ Brett A. Bednarcyk ${ }^{3}$ and Evan J. Pineda ${ }^{4}$ \\ NASA Glenn Research Center, Cleveland, OH, 44135, United States of America
}

\begin{abstract}
Three-dimensional (3D) woven composites are an attractive means of achieving superior mechanical performance in aerospace structures. Limited analysis capability currently exists to predict both effective elastic and strength properties for these complex composites. In this study, a comparison of three modeling strategies was performed to assess the ability of the different methods to predict the effective elastic properties of four distinct 3D orthogonal woven composites. Two finite element techniques (in-plane and triply-periodic boundary conditions) and one method of cells technique, the Multiscale Generalized Method of Cells, were considered.
\end{abstract}

\section{Introduction}

Three-dimensional (3D) woven composites are increasingly being considered for implementation into aerospace structures due to their enhanced performance (e.g., delamination resistance) over traditional laminated composites. However, limited analysis capability exists to predict properties required for large structural models. A significant portion of the literature focuses on using analytical or finite element (FE) techniques to explicitly model a 3D woven architecture (e.g., Refs. [1-5]). This study explores three techniques for predicting effective properties of 3D orthogonal woven composites.

Determining effective properties of heterogeneous materials is a key goal of all micromechanics techniques. As such, numerous methods have been developed to calculate these properties based on constituent material properties and geometric arrangements (i.e., perform a homogenization procedure). The principle of scale separation is essential to determining effective properties and assessing their application to a problem of interest [6]. This principle states that a characteristic microscale length (i.e., individual constituents) should be much smaller than that of the macroscale (i.e., homogenized). In such a scenario, macroscale applied loadings are assumed to be constant over some microscale volume and uninfluenced by external boundaries. Triply-periodic (TP) boundary conditions (BCs) are commonly implemented in homogenization approaches to remove the influence of any external boundaries. However, these BCs may not be appropriate for 3D woven composites since periodicity in the through-thickness direction often does not exist.

One key challenge in modeling 3D woven composites is addressing the issue of scale separation. For these composites, the repeating unit cell (RUC) geometry is significantly larger than that of traditional composite materials. This size differential can easily lead to a scenario where effective properties determined from a particular "microscale" volume are used over a similarly sized macroscale volume (e.g., in a FE model of a large structure). Should these

\footnotetext{
${ }^{1}$ Research Aerospace Engineer, Multiscale and Multiphysics Modeling Branch, AIAA Member.

${ }^{2}$ Aerospace Engineer, Mechanical Systems Analysis and Simulation Branch.

${ }^{3}$ Research and Development Engineer, Multiscale and Multiphysics Modeling Branch, AIAA Associate Fellow.

${ }^{4}$ Research Aerospace Engineer, Multiscale and Multiphysics Modeling Branch, AIAA Senior Member.
} 
volumes become similar, either nonlocal constitutive relationships should be employed or the woven architecture should be explicitly simulated. Despite this limitation, it is common for hierarchical multiscale modeling approaches to use homogenized effective properties. This challenge remains to be fully explored.

In this work, three modeling strategies are explored as a means to estimate effective properties of 3D orthogonal woven composites. Both FE and the Multiscale Generalized Method of Cells (MSGMC) approaches are considered. The connection between material properties determined using such techniques and their incorporation into larger scale structural analyses is a topic of current research.

\section{Analysis Methods}

In this section, the modeling approaches used to determine effective properties of 3D orthogonal woven composites are described.

\section{A. Finite Element}

FE approaches are commonly used to calculate effective properties of heterogeneous composites due to their wide availability, ease of understanding, and accuracy provided an appropriate mesh is generated. In this work, DigimatFE [7] was used to create the geometries, predict yarn properties, and generate FE meshes of 3D woven composites. Abaqus [8] was used as the FE solver. Scripts were developed in order to implement TP BCs and calculate effective properties from simulation results. These BCs, consistent with Stier et al. [9], were implemented in Abaqus for a parallelpiped mesh of a unit cell. In general, this requires specifying a series of displacement constraint equations on individual surface nodes in the model. Three Abaqus reference points were created and are used to impose unique BCs. Displacements, consistent with a prescribed average strain level, were applied at these reference points and used to calculate the effective stiffness matrix of the composite.

Consider a heterogeneous material comprised of linearly elastic, orthotropic constituents only. The principles of local action and scale separation indicate that a uniform applied average strain state $(\bar{\varepsilon})$ produces a uniform average stress state $(\overline{\boldsymbol{\sigma}})$. The stresses and strains are related to each other through the average stiffness matrix $(\overline{\boldsymbol{C}})$ as shown in Eq. (1).

$$
\left[\begin{array}{l}
\bar{\sigma}_{11} \\
\bar{\sigma}_{22} \\
\bar{\sigma}_{33} \\
\bar{\sigma}_{12} \\
\bar{\sigma}_{13} \\
\bar{\sigma}_{23}
\end{array}\right]=\left[\begin{array}{cccccc}
\bar{C}_{11} & \bar{C}_{12} & \bar{C}_{13} & 0 & 0 & 0 \\
\bar{C}_{12} & \bar{C}_{22} & \bar{C}_{23} & 0 & 0 & 0 \\
\bar{C}_{13} & \bar{C}_{23} & \bar{C}_{33} & 0 & 0 & 0 \\
0 & 0 & 0 & \bar{C}_{44} & 0 & 0 \\
0 & 0 & 0 & 0 & \bar{C}_{55} & 0 \\
0 & 0 & 0 & 0 & 0 & \bar{C}_{66}
\end{array}\right]\left[\begin{array}{c}
\bar{\varepsilon}_{11} \\
\bar{\varepsilon}_{22} \\
\bar{\varepsilon}_{33} \\
2 \bar{\varepsilon}_{12} \\
2 \bar{\varepsilon}_{13} \\
2 \bar{\varepsilon}_{23}
\end{array}\right]
$$

In practice, both $\overline{\boldsymbol{\sigma}}$ and $\overline{\boldsymbol{\varepsilon}}$ can be determined by volume averaging local quantities (e.g., over elements). $\overline{\boldsymbol{C}}$ is inverted to give the average compliance matrix, $\overline{\boldsymbol{S}}$. Nine effective constants can then be defined using the following relationships:

$$
\begin{gathered}
\bar{E}_{11}=1 / \bar{S}_{11} \\
\bar{E}_{22}=1 / \bar{S}_{22} \\
\bar{E}_{33}=1 / \bar{S}_{33} \\
\bar{v}_{12}=-\bar{S}_{21} / \bar{S}_{11} \\
\bar{v}_{13}=-\bar{S}_{31} / \bar{S}_{11} \\
\bar{v}_{23}=-\bar{S}_{32} / \bar{S}_{22} \\
\bar{G}_{12}=1 / \bar{S}_{44} \\
\bar{G}_{13}=1 / \bar{S}_{55} \\
\bar{G}_{23}=1 / \bar{S}_{66}
\end{gathered}
$$

where $\bar{E}$ and $\bar{G}$ are effective longitudinal and shear moduli respectively, and $\bar{v}$ are effective Poisson ratios. 
In order to determine $\overline{\boldsymbol{C}}$ for the composite, a series of analyses are performed by independently varying the components of $\overline{\boldsymbol{\varepsilon}}$ and volume averaging the resulting element stresses. For example, if $\bar{\varepsilon}_{11}=1$ and all other strain components are set to zero, the volume-averaged stress in the composite is equal to the first column of the stiffness matrix. Note that the applied displacements can be scaled to a smaller value for numerical stability, but this scaling factor must be accounted for when establishing the stiffness tensor. As a consequence of using periodic BCs, the volume averaged strain will approximately correspond to the applied strain and can be used to verify correct model implementation.

A Python script was developed to volume-averaged element stresses and strains by utilizing the Abaqus Python Application Programming Interface (API). By default, Abaqus outputs field quantities in the material coordinate system. A coordinate transformation was first performed in order to rotate the stress and strain tensors into the global coordinate system. Volume-averaged strains and stresses were then determined using the following equations:

$$
\begin{aligned}
& \overline{\boldsymbol{\varepsilon}}=\frac{1}{a b c} \sum_{i=1}^{\text {Nelms }} \sum_{j=1}^{\text {Nint }} \operatorname{IVOL}_{i, j} \boldsymbol{\varepsilon} \\
& \overline{\boldsymbol{\sigma}}=\frac{1}{a b c} \sum_{i=1}^{\text {Nelms }} \sum_{j=1}^{\text {Nint }_{i}} \operatorname{IVOL}_{i, j} \boldsymbol{\sigma}
\end{aligned}
$$

where Nelms is the number of elements in the mesh, Nint $_{i}$ is the number of integration points for the $i^{\text {th }}$ element, $I V O L_{i, j}$ is the volume associated with the $j^{\text {th }}$ integration point in the $i^{\text {th }}$ element (an Abaqus output variable), and $\boldsymbol{\varepsilon}$ and $\boldsymbol{\sigma}$ are the integration point strain and stress tensors, respectively, in the global coordinate system. Effective properties for the composite can then be calculated using the relationships in Eq. (2).

3D woven composites are often comprised of a single ply. As a result, the use of periodicity in the thickness direction may lead to different material property predictions. Additional simulations were performed that utilized inplane periodic (IPP) BCs only. Unlike the TP BCs, IPP BCs allow faces normal to the thickness direction to be free and to independently expand/ contract. Digimat-FE [7] was used to both impose these boundary conditions and calculate effective in-plane material parameters for the composite. For a given geometry, the influence of the external boundaries in the thickness direction may not be negligible. Hence, these predicted material parameters may or may not be consistent with the actual material properties (i.e., those uninfluenced by external boundaries).

\section{B. Multiscale Generalized Method of Cells}

The Generalized Method of Cells (GMC) micromechanics theory is an efficient, semi-analytical method that provides the homogenized, nonlinear constitutive response of a composite material. Its foundations for single scale analysis, along with validation of its results, are well-established in the literature (cf., Ref. [10]). The GMC method considers the composite microstructure, on a given length scale, to be periodic, with an RUC as shown (at a given length scale) in Fig. 1. The unit cell is discretized into $N_{\alpha} \times N_{\beta} \times N_{\gamma}$ subcells, each of which may contain a distinct material. However, as indicated in Fig. 1, the unique feature of the MSGMC is that the materials occupying the subcells on a given length scale may themselves be heterogeneous composite materials, represented by a unique RUC. A given analysis may consist of $k$ arbitrary explicit length scales (see Fig. 1). The highest length scale considered is denoted as Level 0 , whereas, the current length scale under consideration is length scale $i$, where $i=0,1, \ldots, k$. 


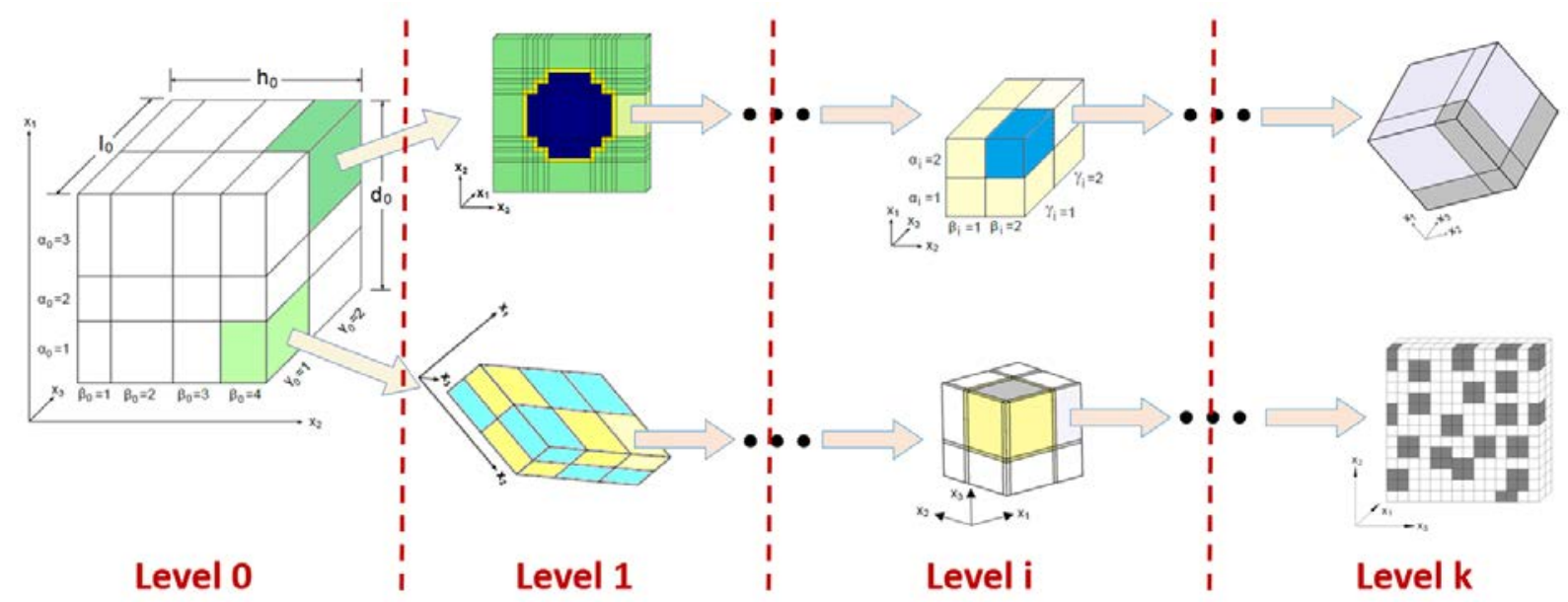

Fig. 1 MSGMC repeating unit cells (RUCs) and subcells across an arbitrary number of length scales.

The GMC theory assumes a first-order displacement field in the subcells at a given scale, resulting in constant stresses and strains per subcell [10]. Assuming infinitesimal strains, the constitutive equation for the subcells at Level $i$ is given by,

$$
\boldsymbol{\sigma}_{i}^{\left(\alpha_{i} \beta_{i} \gamma_{i}\right)}=\boldsymbol{C}_{i}^{\left(\alpha_{i} \beta_{i} \gamma_{i}\right)} \boldsymbol{\varepsilon}_{i}^{\left(\alpha_{i} \beta_{i} \gamma_{i}\right)}
$$

where $\sigma_{i}$ is the stress tensor, $\mathbf{C}_{i}$ is the stiffness tensor, and $\boldsymbol{\varepsilon}_{i}$ is the total strain tensor for the $\mathrm{i}^{\text {th }}$ Level. The superscript $\left(\alpha_{i} \beta_{i} \gamma_{i}\right)$ denotes the particular subcell at Level $i$. Note that inelastic and thermal strains are not considered herein. Satisfaction of displacement and traction continuity between subcells in an average (integral) sense, and imposition of periodicity conditions along the RUC boundaries enable the establishment of a system of linear algebraic equations, which can be solved to determine the elastic strain concentration tensors, $\boldsymbol{A}_{i}^{\left(\alpha_{i} \beta_{i} \gamma_{i}\right)}$. The reader is referred to Aboudi et al. [10] for more details regarding this formulation. At a given level (i.e., scale), $i$, these concentration tensors characterize the local strain tensors in the subcells in terms of the RUC-averaged total strain tensor, $\overline{\boldsymbol{\varepsilon}}_{i}$, i.e.,

$$
\boldsymbol{\varepsilon}_{i}^{\left(\alpha_{i} \beta_{i} \gamma_{i}\right)}=\boldsymbol{A}_{i}^{\left(\alpha_{i} \beta_{i} \gamma_{i}\right)} \overline{\boldsymbol{\varepsilon}}_{i}
$$

In a multiscale analysis, all terms in Eq. (5) depend on the location of the Level $i$ RUC within all higher scale RUCs. In essence, the strain in a given subcell at Level $k$ depends on the path taken down the length scales from Level 0. Eq. (4) can be recast in terms of average strains by substituting Eq. (5) into Eq. (4),

$$
\boldsymbol{\sigma}_{i}^{\left(\alpha_{i} \beta_{i} \gamma_{i}\right)}=\boldsymbol{C}_{i}^{\left(\alpha_{i} \beta_{i} \gamma_{i}\right)} \boldsymbol{A}_{i}^{\left(\alpha_{i} \beta_{i} \gamma_{i}\right)} \overline{\boldsymbol{\varepsilon}}_{i}
$$

The RUC-averaged stress tensor is given by,

$$
\overline{\boldsymbol{\sigma}}_{i}=\frac{1}{d_{i} h_{i} l_{i}} \sum_{\alpha_{i}=1}^{N_{\alpha_{i}}} \sum_{\beta_{i}=1}^{N_{\beta_{i}}} \sum_{\gamma_{i}=1}^{N_{\gamma_{i}}} d_{\alpha_{i}} h_{\beta_{i}} l_{\gamma_{i}} \boldsymbol{\sigma}_{i}^{\left(\alpha_{i} \beta_{i} \gamma_{i}\right)}
$$

where $d_{\alpha_{i}}, h_{\beta_{i}}$, and $l_{\gamma_{i}}$ are the dimensions of subcell $\left(\alpha_{i} \beta_{i} \gamma_{i}\right)$ and $d_{i}, h_{i}$, and $l_{i}$ are the RUC dimensions for Level $i$. Eq. (6) and Eq. (7) lead to,

$$
\overline{\boldsymbol{\sigma}}_{i}=\frac{1}{d_{i} h_{i} l_{i}} \sum_{\alpha_{i}=1}^{N_{\alpha_{i}}} \sum_{\beta_{i}=1}^{N_{\beta_{i}}} \sum_{\gamma_{i}=1}^{N_{\gamma_{i}}} d_{\alpha_{i}} h_{\beta_{i}} l_{\gamma_{i}} \boldsymbol{C}_{i}^{\left(\alpha_{i} \beta_{i} \gamma_{i}\right)} \boldsymbol{A}_{i}^{\left(\alpha_{i} \beta_{i} \gamma_{i}\right)} \overline{\boldsymbol{\varepsilon}}_{i}
$$

The effective constitutive equation at Level $i$ is given by, 


$$
\overline{\boldsymbol{\sigma}}_{i}=\boldsymbol{C}_{i}^{*} \overline{\boldsymbol{\varepsilon}}_{i}
$$

A comparison of Eq. (8) and Eq. (9) indicates that the effective stiffness tensor, $\boldsymbol{C}_{i}^{*}$, at Level $i$ is given by,

$$
\boldsymbol{C}_{i}^{*}=\frac{1}{d_{i} h_{i} l_{i}} \sum_{\alpha_{i}=1}^{N_{\alpha_{i}}} \sum_{\beta_{i}=1}^{N_{\beta_{i}}} \sum_{\gamma_{i}=1}^{N_{\gamma_{i}}} d_{\alpha_{i}} h_{\beta_{i}} l_{\gamma_{i}} \boldsymbol{C}_{i}^{\left(\alpha_{i} \beta_{i} \gamma_{i}\right)} \boldsymbol{A}_{i}^{\left(\alpha_{i} \beta_{i} \gamma_{i}\right)}
$$

In the MSGMC, the scales are linked by considering the RUC-averaged stress, strain, and stiffness tensors at scale $i$ to be equal to the local subcell stress, strain, and stiffness tensors of the applicable subcell from the next higher length scale (i-1). An appropriate coordinate transformation is used to account for the potential coordinate system change from scale to scale. That is,

$$
\overline{\boldsymbol{\varepsilon}}_{i}=\mathbf{T}_{2}^{i} \boldsymbol{\varepsilon}_{i-1}^{\left(\alpha_{i-1} \beta_{i-1} \gamma_{i-1}\right)}, \overline{\boldsymbol{\sigma}}_{i}=\mathbf{T}_{2}^{i} \boldsymbol{\sigma}_{i-1}^{\left(\alpha_{i-1} \beta_{i-1} \gamma_{i-1}\right)}, \mathbf{C}_{i}^{*}=\mathbf{T}_{4}^{i} \mathbf{C}_{i-1}^{\left(\alpha_{i-1} \beta_{i-1} \gamma_{i-1}\right)}
$$

where $\boldsymbol{T}_{2}^{i}$ and $\boldsymbol{T}_{4}^{i}$ are the appropriate second and fourth-order coordinate transformation tensors, respectively. Hence, starting with the lowest scale ( $k$ ) RUC (see Fig. 1), whose subcells contain only monolithic materials, the effective stiffness tensor can be calculated using the standard GMC method. This stiffness tensor (after an appropriate coordinate transformation) then represents the homogenized material in one of the subcells within an RUC at the next higher length scale $(k-1)$. Given the transformed effective stiffness tensors of all subcells at the successively higher length scale, the effective stiffness tensor of the RUC at this Level can be determined. This stiffness tensor can then be transformed and passed along to the next higher length scale in a similar fashion, and the process repeats until the highest length scale considered $(i=0)$ is reached. As an example, for an MSGMC analysis considering three length scales ( $i=0,1$, and 2), the overall effective stiffness tensor can be written using Eq. (10) and Eq. (11) as,

$$
\mathbf{C}_{0}^{*}=\frac{1}{d_{0} h_{0} l_{0}} \sum_{\alpha_{0} \beta_{0} \gamma_{0}} d_{\alpha_{0}} h_{\beta_{0}} l_{\gamma_{0}}\left\{\frac{\left(\mathbf{T}_{4}^{1}\right)^{-1}}{d_{1} h_{1} l_{1}} \sum_{\alpha_{1} \beta_{1} \gamma_{1}} d_{\alpha_{1}} h_{\beta_{1}} l_{\gamma_{1}}\left[\frac{\left(\mathbf{T}_{4}^{2}\right)^{-1}}{d_{2} h_{2} l_{2}} \sum_{\alpha_{2} \beta_{2} \gamma_{2}} d_{\alpha_{2}} h_{\beta_{2}} l_{\gamma_{2}} \mathbf{C}_{2}^{\left(\alpha_{2} \beta_{2} \gamma_{2}\right)} \mathbf{A}_{2}^{\left(\alpha_{2} \beta_{2} \gamma_{2}\right)}\right]^{\left(\alpha_{1} \beta_{\beta} \gamma_{1}\right)} \mathbf{A}_{1}^{\left(\alpha_{1} \beta_{\gamma_{1}}\right)}\right\}^{\left(\alpha_{0} \beta_{0} \gamma_{0}\right)} \mathbf{A}_{0}^{\left(\alpha_{0} \beta_{0} \gamma_{0}\right)}
$$

where a contracted notation has been used for the triple summation at each scale. Note that, in Eq. (11), the superscript on the bracketed terms indicates that all variables within the brackets are a function of the subcell indices from the next higher length scale (including lower scale dimensions and subcell indices). The intent of this notation is to fully define the location of a subcell at a given scale as one progresses down the length scales. For example, using this notation, the effective stiffness tensor at Level 2, from Eq. (11) can be written as,

$$
\left\{\left[\mathbf{C}_{2}^{*}\right]^{\left(\alpha_{1} \beta_{1} \gamma_{1}\right)}\right\}^{\left(\alpha_{0} \beta_{0} \gamma_{0}\right)}=\left\{\left[\mathbf{T}_{4}^{2}\right]^{\left(\alpha_{1} \beta_{1} \gamma_{1}\right)}\right\}^{\left(\alpha_{0} \beta_{0} \gamma_{0}\right)}\left\{\mathbf{C}_{1}^{\left(\alpha_{1} \beta_{1} \gamma_{1}\right)}\right\}^{\left(\alpha_{0} \beta_{0} \gamma_{0}\right)}
$$

as there are distinct $\boldsymbol{C}_{2}^{*}$ values for every Level 1 subcell, while there are distinct Level 1 RUCs present within each Level 0 subcell.

Additionally, MSGMC can perform multiscale localization of the stress and strain tensors. The stress tensor for any subcell at any length scale can be determined through localization relationships [10], or by simply using the strain tensor, along with the constitutive equation, at the appropriate length scale.

In order to develop a model of a 3D woven composite in MSGMC, voxel-based finite element meshes were converted into an appropriate RUC for an MSGMC analysis. A series of Matlab scripts were written to import a finite element mesh (e.g., from TexGen, Abaqus, or MSC Marc), determine unique material orientations, modify the orientations to be consistent with MSGMC definitions, and generate the MSGMC model. A two-step homogenization procedure was used to determine the effective mechanical response for the composite by homogenizing subcells in the thickness direction prior to homogenizing in-plane. This procedure was shown by Bednarcyk and Arnold [11] to improve the prediction of in-plane properties and is typically used in conjunction with the MSGMC. This required defining a number of "stacks" (i.e., single columns of subcells in the thickness direction). An example of these stacks 
for a plain weave fabric is shown in Fig. 2. Duplicate stacks (same material/ orientation distributions) were identified and removed from the model to reduce memory requirements.

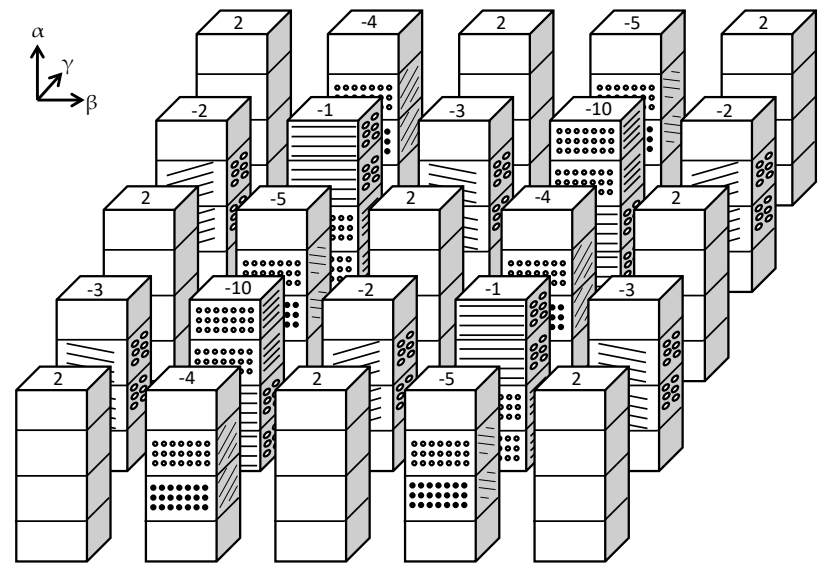

Fig. 2 MSGMC plain weave fabric representation.

\section{Simulation Details}

For comparison purposes, each analysis method was used to predict effective material parameters or properties for four distinct IM7/ RTM6 3D orthogonal woven composite panels. Baseline weave architecture designs were proposed by Bally Ribbon Mills, Inc. Digimat-FE was used to generate an idealized/ simplified woven geometry for each panel. A typical 3D orthogonal geometry is determined by specifying an arrangement of layered, straight warp and weft yarns. One or more linker yarns then are woven through the thickness (Z-direction) of the composite at pre-determined locations. The warp, weft, and through-thickness portion of the linker yarns are designed to be orthogonal to one another.

In this study, two woven architectures were considered. Two variants on each architecture were generated by assuming each yarn to be comprised of $6 \mathrm{~K}$ or $12 \mathrm{~K}$ fiber tows. The geometric parameters associated with the warp, weft, and linker yarns, as used in Digimat-FE, are shown in Table 1 for each of the four panel geometries considered in this study. In practice, during the weaving process, yarns are competing for the same space and some overlap may occur. This overlap is difficult to numerically simulate. In order to better match the proposed unit cell sizes, the linker yarns were altered by lowering the filament count (e.g., 1200 instead of 6000) and adjusting the linker yarn dimensions. These yarn modifications, however, were compensated for by increasing the fiber modulus in the axial dimension to match that of the idealized $6 \mathrm{~K}$ or $12 \mathrm{~K}$ yarn. Elastic material properties were obtained from the literature for the transversely isotropic IM7 fiber [12, 13] and isotropic RTM6 matrix [14]. Digimat-FE calculated yarn material properties based on constituent properties, filament count, and tow geometry using a mean-field homogenization technique [7]. The calculated warp, weft, and linker yarn orthotropic elastic constants for each of the four panels are shown in Table 2. 
Table 1 Yarn Parameters

\begin{tabular}{|c|c|c|c|}
\hline \multicolumn{2}{|c|}{ Parameter } & Panels 1, 3 & Panels 2, 4 \\
\hline \multirow{3}{*}{ Warp } & Filament Count & 6000 & 12000 \\
\cline { 2 - 4 } & Yarn Height (mm) & 0.18 & 0.36 \\
\cline { 2 - 4 } & Yarn Width (mm) & 1.2 & 1.2 \\
\hline \multirow{3}{*}{ Weft } & Filament Count & 6000 & 12000 \\
\cline { 2 - 4 } & Yarn Height (mm) & 0.174 & 0.285 \\
\cline { 2 - 4 } & Yarn Width (mm) & 1.24 & 1.5 \\
\hline \multirow{3}{*}{ Linker } & Filament Count & 1200 & 2200 \\
\cline { 2 - 4 } & Yarn Height (mm) & 0.1 & 0.2 \\
\cline { 2 - 4 } & Yarn Width (mm) & 0.43 & 0.4 \\
\hline \multicolumn{2}{|c|}{ Fiber Diameter ( $\boldsymbol{\mu m}$ ) } & \multicolumn{2}{|c}{5.2} \\
\hline
\end{tabular}

Table 2 Calculated Yarn Material Properties

\begin{tabular}{|c|c|c|c|c|c|c|}
\hline \multirow{2}{*}{ Property } & \multicolumn{2}{|c|}{ Warp Yarn } & \multicolumn{2}{c|}{ Weft Yarn } & \multicolumn{2}{c|}{ Linker Yarn } \\
\cline { 2 - 7 } & Panels 1, 3 & Panels 2, 4 & Panels 1, 3 & Panels 2, 4 & Panels 1, 3 & Panels 2, 4 \\
\hline $\mathbf{E}_{\mathbf{1 1}}$ (MPa) & 208040 & 208040 & 208270 & 210200 & 1027000 & 1079000 \\
\hline $\mathbf{E}_{\mathbf{2 2}}$ (MPa) & 9999 & 9999 & 10016 & 10164 & 10544 & 10295 \\
\hline $\mathbf{E}_{\mathbf{3 3}}$ (MPa) & 9999 & 9999 & 10016 & 10164 & 10544 & 10295 \\
\hline $\mathbf{V}_{\mathbf{1 2}}$ & 0.233 & 0.233 & 0.232 & 0.232 & 0.232 & 0.233 \\
\hline $\mathbf{V}_{\mathbf{1 3}}$ & 0.233 & 0.233 & 0.232 & 0.232 & 0.232 & 0.233 \\
\hline $\mathbf{V}_{\mathbf{2 3}}$ & 0.363 & 0.363 & 0.363 & 0.36 & 0.369 & 0.373 \\
\hline $\mathbf{G}_{\mathbf{1 2}}$ (MPa) & 3707 & 3707 & 3713 & 3770 & 3734 & 3649 \\
\hline $\mathbf{G}_{\mathbf{1 3}} \mathbf{( M P a )}$ & 3707 & 3707 & 3713 & 3770 & 3734 & 3649 \\
\hline $\mathbf{G}_{\mathbf{2 3}} \mathbf{( M P a )}$ & 3668 & 3668 & 3675 & 3737 & 3852 & 3749 \\
\hline
\end{tabular}

Fig. 3(a-b) shows the woven geometry for one 3D woven architecture comprised of idealized yarns with $6 \mathrm{~K}$ (Panel 1) and 12K (Panel 2) fiber tows, respectively. Fig. 4(a-b) contain similar images of a separate architecture represented by Panels 3-4. The Digimat-FE calculated unit cell sizes and fiber volume fractions are given in Table 3. Each geometry was discretized into $140 \times 60 \times 60$ voxels in the warp, weft, and Z directions, respectively, for a total of 504,000 voxels. The resulting discretizations (excluding the matrix) are shown in Fig. 3(c-d) and Fig. 4(c-d). For all FE and MSGMC analyses, each voxel was represented by a single reduced-integration isoparametric brick element and subcell, respectively. One of the challenges associated with simulating 3D woven composites is that voxel-based methods often require a large number of voxels in order to accurately capture the geometry of a relatively large unit cell size (when compared to traditional unidirectional or 2D woven composites). Furthermore, when combined with FE techniques, some non-physical fluctuations in the local fields can occur [15] due to element discontinuities. Although not considered in this study, these fluctuations can significantly influence damage initiation and growth [15]. 

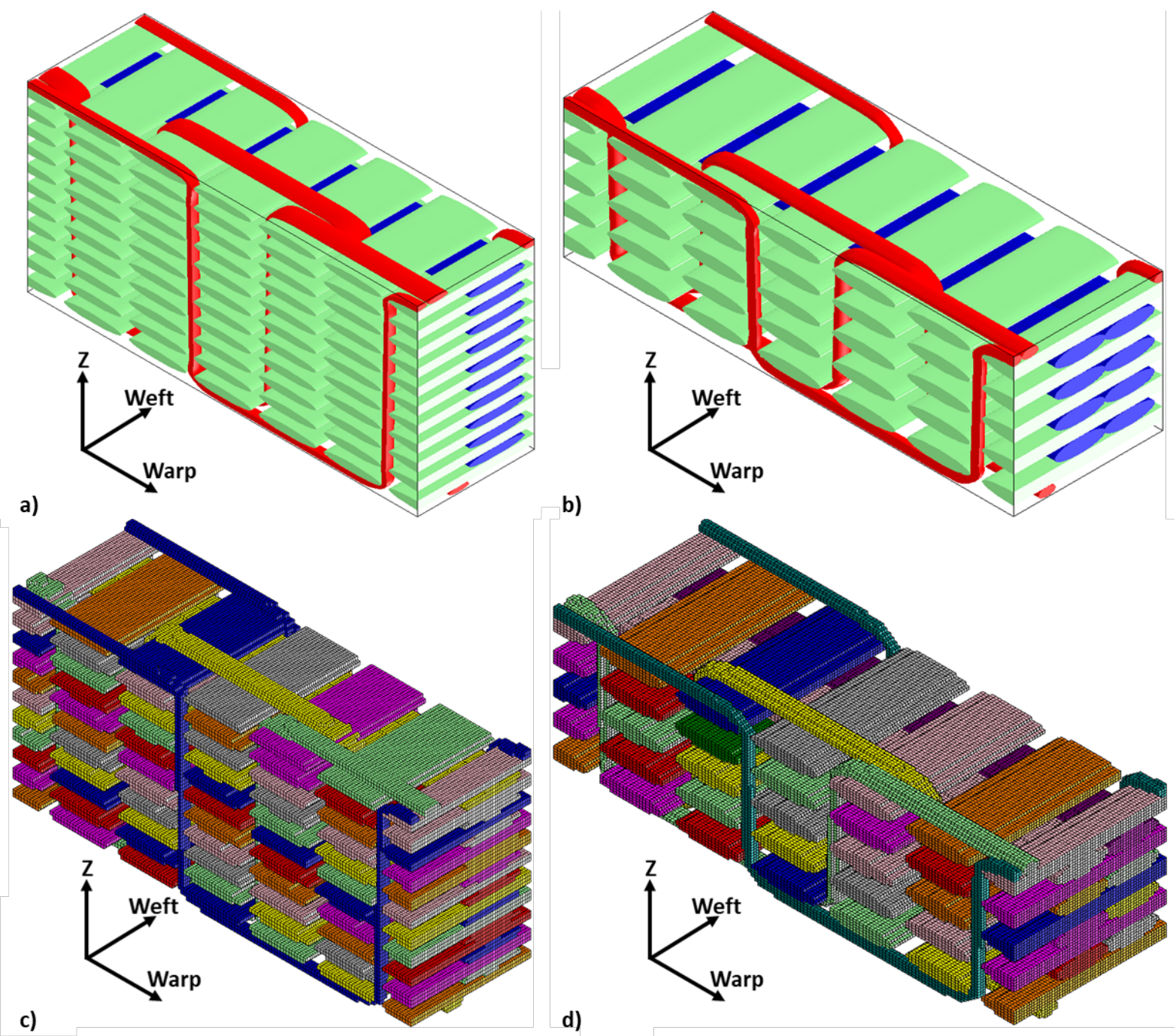

Fig. 3 3D woven geometries for a) Panel 1 and b) Panel 2. Voxelized discretizations for c) Panel 1 and d) Panel 2 (matrix voxels not shown). 

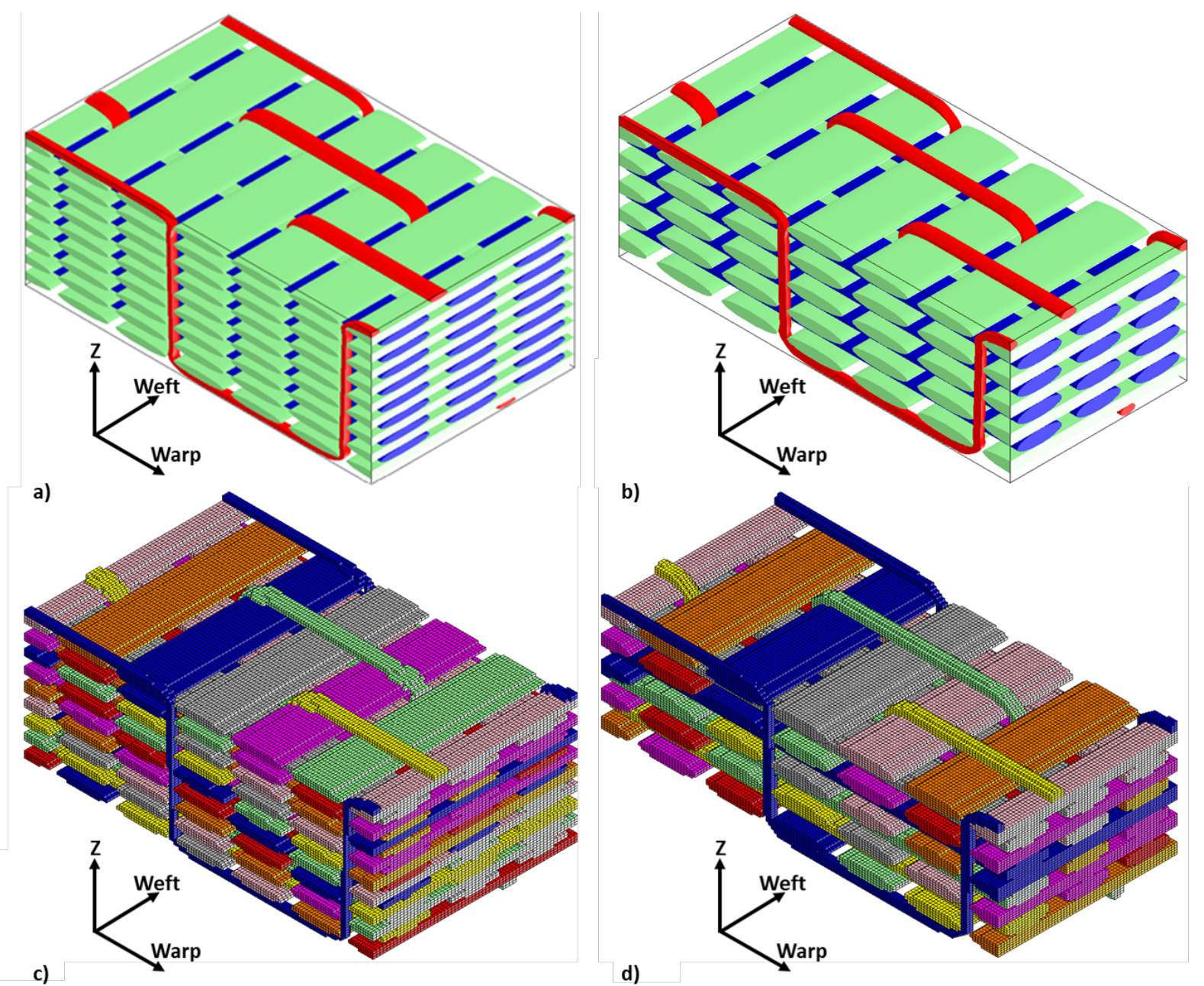

Fig. 4 3D woven geometries for a) Panel 3 and b) Panel 4. Voxelized discretizations for c) Panel 3 and d) Panel 4 (matrix voxels not shown).

Table 3 Geometric and Discretization Parameters

\begin{tabular}{|c|c|c|c|c|c|}
\hline \multicolumn{2}{|c|}{ Parameter } & Panel 1 & Panel 2 & Panel 3 & Panel 4 \\
\hline \multirow{3}{*}{ Unit Cell Size (mm) } & Warp & 8.45 & 10.71 & 8.51 & 10.62 \\
\cline { 2 - 6 } & Weft & 2.54 & 3.55 & 5.00 & 4.80 \\
\cline { 2 - 6 } & Z & 3.91 & 3.30 & 3.24 & 3.30 \\
\hline \multirow{4}{*}{ Number of Voxels } & Warp & \multicolumn{4}{|c|}{140} \\
\cline { 2 - 6 } & Weft & \multicolumn{5}{|c|}{60} \\
\cline { 2 - 6 } & Z & \multicolumn{5}{|c|}{504,000} \\
\cline { 2 - 6 } & Total & \multicolumn{5}{|c|}{0.41} & 0.43 \\
\hline
\end{tabular}




\section{Results}

Predicted effective material parameters/ properties using both FE techniques (IPP and TP BCs) and the MSGMC are shown in Table 4 for each of the four panels. The in-plane properties obtained from using both IPP and TP BCs implemented in a classical FE framework were nearly identical across all panels. This indicates that the difference in periodic BC choice for the FE calculations has a minimal impact on in-plane material properties for the architectures considered in this study. However, the effect on through-thickness properties remains to be explored.

In general, the MSGMC technique yielded consistent predictions for most of the nine elastic constants (excluding $E_{33}$ and several of the out-of-plane Poisson ratios) when compared to the TP FE calculations. For example, the predicted $E_{33}$ for MSGMC was 7-19\% lower than that of the TP FE technique while both in-plane moduli ( $E_{11}$ and $E_{22}$ ) were within $2 \%$. The exact cause of these discrepancies are unknown, but could be due to the lack of normal/ shear coupling inherent to the MSGMC technique [10]. Higher-fidelity method of cells techniques, such as the parametric high-fidelity generalized method of cells [16], would likely attain more agreeable results for all material properties. This topic will be addressed in a future study. A detailed comparison to experimental data is currently in progress.

Table 4 Predicted Material Parameters/ Properties

\begin{tabular}{|c|c|c|c|c|c|c|c|c|c|c|}
\hline \multirow[b]{2}{*}{ Geometry } & \multirow[b]{2}{*}{ Method } & \multicolumn{9}{|c|}{ Material Parameter/ Property } \\
\hline & & $\begin{array}{c}\mathrm{E}_{11} \\
(\mathrm{MPa})\end{array}$ & $\begin{array}{c}E_{22} \\
(\mathrm{MPa})\end{array}$ & $\begin{array}{c}\mathrm{E}_{33} \\
(\mathrm{MPa})\end{array}$ & $\mathbf{v}_{12}$ & $\mathbf{V}_{13}$ & $V_{23}$ & $\begin{array}{c}\mathrm{G}_{12} \\
(\mathrm{MPa})\end{array}$ & $\begin{array}{c}\mathrm{G}_{13} \\
(\mathrm{MPa})\end{array}$ & $\begin{array}{c}\mathrm{G}_{23} \\
(\mathrm{MPa})\end{array}$ \\
\hline \multirow{3}{*}{ Panel 1} & FE (IPP) & 41857 & 72877 & - & 0.027 & - & - & 2201 & - & - \\
\hline & FE (TP) & 41785 & 72851 & 8450 & 0.027 & 0.354 & 0.326 & 2190 & 1891 & 1868 \\
\hline & MSGMC & 43299 & 72788 & 6885 & 0.025 & 0.409 & 0.395 & 2272 & 1777 & 1739 \\
\hline \multirow{3}{*}{ Panel 2} & FE (IPP) & 55073 & 64287 & - & 0.028 & - & - & 2249 & - & - \\
\hline & FE (TP) & 54971 & 64245 & 7707 & 0.028 & 0.402 & 0.368 & 2238 & 1944 & 1947 \\
\hline & MSGMC & 55869 & 64410 & 6965 & 0.028 & 0.406 & 0.401 & 2360 & 1801 & 1789 \\
\hline \multirow{3}{*}{ Panel 3} & FE (IPP) & 57617 & 72613 & - & 0.026 & - & - & 2378 & - & - \\
\hline & FE (TP) & 57580 & 72592 & 7883 & 0.026 & 0.388 & 0.369 & 2365 & 2047 & 2045 \\
\hline & MSGMC & 58381 & 72691 & 7278 & 0.026 & 0.400 & 0.394 & 2456 & 1918 & 1899 \\
\hline \multirow{3}{*}{ Panel 4} & FE (IPP) & 58440 & 64817 & - & 0.027 & - & - & 2264 & - & - \\
\hline & FE (TP) & 58367 & 64798 & 7593 & 0.027 & 0.408 & 0.378 & 2252 & 1991 & 1978 \\
\hline & MSGMC & 59138 & 65089 & 7053 & 0.028 & 0.404 & 0.401 & 2400 & 1835 & 1824 \\
\hline
\end{tabular}

\section{Summary and Conclusions}

Three-dimensional (3D) woven composites architectures present unique challenges when trying to predict material properties. In this study, two different methods were evaluated for predicting effective material properties: a finite element (FE) technique using triply-periodic (TP) boundary conditions (BCs) and the Multiscale Generalized Method of Cells (MSGMC) technique. A separate FE technique using in-plane periodic BCs (independent expansion/ contraction allowed on opposite faces in thickness direction) was used to predict in-plane effective material parameters. Four separate 3D orthogonal woven geometries were considered. Both FE techniques yielded consistent in-plane effective properties suggesting that the periodicity assumption in the thickness direction did not significantly influence the in-plane properties. However, the effect on out-of-plane properties remains to be explored. The MSGMC technique yielded consistent predictions for in-plane material properties with the FE results while some discrepancies were observed in the out-of-plane property predictions. While effective property estimates for all methods considered 
in this study were in general agreement, the incorporation of such properties for 3D woven composites into higherlevel structural models remains an issue to be addressed.

\section{Acknowledgments}

This work was performed as part of NASA's Composite Technology for Exploration Project. The authors also appreciate 3D weave design support provided by Mr. Hakan Gokce of Bally Ribbon Mills, Inc.

\section{References}

[1] Green, S., M. Matveev, A. Long, D. Ivanov, and S. Hallett, "Mechanical modelling of 3D woven composites considering realistic unit cell geometry," Composite Structures, Vol. 118, 2014, pp. 284-293. doi: 10.1016/j.compstruct.2014.07.005

[2] Tsukrov, I., et al., "Finite element modeling to predict cure-induced microcracking in three-dimensional woven composites," International Journal of Fracture, Vol. 172, No. 2, 2011, pp. 209-216. doi: 10.1007/s10704-011-9659-X

[3] Tan, P., L. Tong, and G. Steven, "Behavior of 3D orthogonal woven CFRP composites. Part II. FEA and analytical modeling approaches," Composites Part A: Applied Science and Manufacturing, Vol. 31, No. 3, 2000, pp. 273-281. doi: 10.1016/S1359-835X(99)00071-8

[4] Tan, P., L. Tong, and G.P. Steven, "Micromechanics models for mechanical and thermomechanical properties of 3D through-the-thickness angle interlock woven composites," Composites Part A: Applied Science and Manufacturing, Vol. 30, No. 5, 1999, pp. 637-648.

[5] Bahei-El-Din, Y., A. Rajendran, and M. Zikry, "A micromechanical model for damage progression in woven composite systems," International Journal of Solids and Structures, Vol. 41, No. 9-10, 2004, pp. 2307-2330. doi: 10.1016/j.ijsolstr.2003.12.006

[6] Geers, M.G.D., V.G. Kouznetseva, and W.A.M. Brekelmans, "Multi-scale computational homogenization: Trends and challenges," Journal of Computational and Applied Mathematics, Vol. 234, 2010, pp. $2175-2182$. doi: 10.1016/j.cam.2009.08.077

[7] Digimat, 2018. Available from: http://www.e-xstream.com/products/digimat/about-digimat.

[8] Abaqus Unified FEA, 2018. Available from: $\underline{\text { https://www.3ds.com/products- }}$ services/simulia/products/abaqus/.

[9] Stier, B., B.A. Bednarcyk, J.W. Simon, and S. Reese, "Investigation of micro-scale architectural effects on damage of composites," NASA/TM-2015-218750, 2015.

[10] Aboudi, J., S.M. Arnold, and B.A. Bednarcyk, Micromechanics of composite materials. Elsevier, Oxford, UK, 2012.

[11] Bednarcyk, B.A. and S.M. Arnold, "Micromechanics-based modeling of woven polymer matrix composites," AIAA Journal, Vol. 41, No. 9, 2003, pp. 1788-1796. doi: 10.2514/2.7297

[12] Autodesk Helius Composite, 2018. Available from: https://www.autodesk.com/products/heliuscomposite/overview.

[13] Hextow IM7 carbon fiber product data sheet, 2018. Available from: https://www.hexcel.com/user_area/content_media/raw/IM7_HexTow_DataSheet.pdf.

[14] Brauner, C., S. Bauer, and A.S. Herrmann, "Analysing process-induced deformation and stresses using a simulated manufacturing process for composite multispar flaps," Journal of Composite Materials, Vol. 49, No. 4, 2015, pp. 387-402. doi: 10.1177/2F0021998313519281

[15] Doitrand, A., C. Fagiano, F.-X. Irisarri, and M. Hirsekorn, "Comparison between voxel and consistent mesoscale models of woven composites," Composites Part A: Applied Science and Manufacturing, Vol. 73, 2015, pp. 143-154. doi: 10.1016/j.compositesa.2015.02.022

[16] Haj-Ali, R. and J. Aboudi, "A new and general formulation of the parametric HFGMC micromechanical method for two and three-dimensional multi-phase composites," International Journal of Solids and Structures, Vol. 50, 2013, pp. 907-919. doi: 10.1016/j.ijsolstr.2012.11.009 